\title{
Article \\ Contrasting Cryptocurrencies with Other Assets: Full Distributions and the COVID Impact
}

\author{
Esfandiar Maasoumi * and Xi Wu \\ Department of Economics, Emory University, Atlanta, GA 30322, USA; xi.wu@emory.edu \\ * Correspondence: emaasou@emory.edu
}

\begin{abstract}
We investigate any similarity and dependence based on the full distributions of cryptocurrency assets, stock indices and industry groups. We characterize full distributions with entropies to account for higher moments and non-Gaussianity of returns. Divergence and distance between distributions are measured by metric entropies, and are rigorously tested for statistical significance. We assess the stationarity and normality of assets, as well as the basic statistics of cryptocurrencies and traditional asset indices, before and after the COVID-19 pandemic outbreak. These assessments are not subjected to possible misspecifications of conditional time series models which are also examined for their own interests. We find that the NASDAQ daily return has the most similar density and co-dependence with Bitcoin daily return, generally, but after the COVID-19 outbreak in early 2020, even S\&P500 daily return distribution is statistically closely dependent on, and indifferent from Bitcoin daily return. All asset distances have declined by 75\% or more after the COVID-19 outbreak. We also find that the highest similarity before the COVID-19 outbreak is between Bitcoin and Coal, Steel and Mining industries, and after the COVID-19 outbreak is between Bitcoin and Business Supplies, Utilities, Tobacco Products and Restaurants, Hotels, Motels industries, compared to several others. This study shed light on examining distribution similarity and co-dependence between cryptocurrencies and other asset classes.
\end{abstract}

Citation: Maasoumi, Esfandiar, and Xi Wu. 2021. Contrasting Cryptocurrencies with Other Assets: Full Distributions and the COVID Impact. Journal of Risk and Financial Management 14: 440. https:// doi.org/10.3390/jrfm14090440

Academic Editor: Shigeyuki Hamori

Received: 11 August 2021

Accepted: 6 September 2021

Published: 14 September 2021

Publisher's Note: MDPI stays neutral with regard to jurisdictional claims in published maps and institutional affiliations.

Copyright: (c) 2021 by the authors. Licensee MDPI, Basel, Switzerland. This article is an open access article distributed under the terms and conditions of the Creative Commons Attribution (CC BY) license (https:// creativecommons.org/licenses/by/ $4.0 /)$.

\section{Introduction}

Since the emergence of Bitcoin based on blockchain technology in 2018, global financial markets have witnessed the birth and rapid rise of cryptocurrencies (cryptos) as a new asset class. Cryptos are based on fundamentally new technologies, the potential of which highly anticipated but not fully understood. In their current form, however, cryptos are also behaving like high growth assets. The cryptocurrency market is an important part of the global assets markets. As of September 2020, there were over 18.53 million Bitcoins in circulation with a total market value of around USD 199.62 billion.

With the rapid development of the cryptocurrency market, the literature has focused on statistical properties and risk behavior of cryptocurrency in comparison with classical assets, like equities and exchange rates. In the setting of time series models, Pichl and Kaizoji (2017) found that cryptocurrency markets are even more volatile than foreign exchange markets. Bouri et al. (2017), (Katsiampa 2017), (Bariviera 2017) and ( Stavroyiannis 2018) observed the phenomenon of volatility clustering in cryptocurrency market. Regimeswitching behaviors are detected by (Bariviera et al. 2017), (Balcombe and Fraser 2017), (Thies and Molnar 2018) have identified structural breaks in the volatility process of Bitcoin via a Bayesian framework. Lahmiri et al. (2019) and (Lahmiri and Bekiros 2018) have pointed out that Bitcoin markets are characterized by long memory and multifractality. Recent studies also examined the performance of cryptos under the COVID-19 pandemic. Vukovic et al. (2021) developed a unique COVID-19 global composite index that measures COVID-19 pandemic time-variant movements on each day. Sarkodie et al. (2021) investigated the implication of COVID-19 outcomes on market prices of several leading cryptos. 
Naeem et al. (2021) quantified the spillover effects among seven cryptos to explore the spillover characteristics cryptos, and discovered that Bitcoin, Litecoin, and Ripple are the dominant transmitters to return spillover. These studies inspired us to investigate how the density similarities between cryptos, stocks and industry groups will be affected by the COVID-19 outbreak.

Statistical similarity and co-dependence are central to the analysis of market efficiency and allocation. Most existing studies focus on Bitcoin returns and "correlation" analysis. For example, (Baur et al. 2018) show that Bitcoin returns are essentially uncorrelated with traditional asset classes such as stocks and bonds, which points to diversification possibilities. Other studies investigate the determinants of Bitcoin returns. Li and Wang (2017) suggest that measures of financial and macroeconomic activity are drivers of Bitcoin returns. Kristoufek (2015) considers financial uncertainty, Bitcoin trading volume in Chinese Yuan and Google trends as potential drivers of Bitcoin returns. Recently, many studies examine whether Bitcoin belongs to any existing asset classes, with many comparing it to gold, others to precious metals or to speculative assets (Baur et al. 2018). Some have classified Bitcoin as a new asset class within currency and commodity groups (Dyhrberg 2016).

Another area of interest is forecasting Bitcoin volatility, since such forecasts represent an important ingredient in risk assessment and allocation, and derivatives pricing theory. Balcilar et al. (2017) analyze the causal relation between trading volume and Bitcoin returns and volatility. They find that volume cannot help to predict the volatility of Bitcoin returns. Bouri et al. (2017) find no evidence for asymmetry in the conditional volatility of Bitcoins when considering the post December 2013 period and investigate the relation between the VIX index and Bitcoin volatility.Al-Khazali et al. (2018) consider a model for daily Bitcoin returns and show that Bitcoin volatility tends to decrease in response to positive news about the US economy.

Scant attention has been paid to the full distributions of these assets. An exception is (Osterrieder and Lorenz 2017) and (Begusic et al. 2018) who have studied the unconditional distribution of Bitcoin returns and found that it has more probability mass in the tails than that of foreign exchange and stock market returns. Findings that are based on models of return and volatility, possibly with conditional covariates, are in effect assessing if similar mechanisms apply to different asset class returns. While this is an aspect of similarity, it does not respond, and indeed may impinge on the assessment of similarity of return outcomes/distributions. Similar distributions may arise from different evolutions and mechanisms over time.

Our objective in this paper is to revisit some stylized facts of cryptocurrency markets and employ econometrics models for accurate volatility forecasts. In contrast to previous studies that use time series models to forecast crypto returns, in this paper we use entropy profiles of different asset classes and indices, as well as the cryptos. We test for similarity between cryptocurrency and stock returns in a manner that captures nonlinearities and higher moments, nonparametrically. We consider both Bitcoin and Ethereum as leading crypto which have large volume and relatively long histories. We use nonparametric entropy metrics to test equality between crypto density and stock market index returns. Time series models (ARIMA and GARCH), in contrast, impose a (traditionally) restrictive linear structure on the return data. This may produce non robust inferences and conclusions.

Efficient market analysis is based on (typically) linear relation between a given asset and market returns. In this paper, we examine the general definition of dependence between crypto return and stock market returns. Stochastic independence is tested and degree of dependence is measured with entropy metrics.

The rest of the paper is organized as follows: Section 2 presents the data analysis and some stylized facts. In Section 3, we calculate nonparametric entropy metrics to test the density equality between two cryptos (Bitcoin and Ethereum), two stock market indexes (S\&P500 and NASDAQ) and 30 commodity industry groups. We conduct equality tests on both marginal distributions and conditional distributions for two periods (preCOVID and COVID era) and compare the results. In Section 4, we consider a Diff-in-diff 
analogy to identify any impact of COVID-19. It is found to be large and significant, producing far greater convergence between asset classes and cryptos. Section 5 provides the concluding remarks.

\section{Data and Basic Characteristics}

The cryptocurrency data and stock market index data set consists of daily spot exchange rates in units of US dollars are from Yahoo Finance ${ }^{1}$. The price observations of Bitcoin (BTC-USD), Ethereum (ETH-USD), S\&P500 stock market index ('GSPC) and NASDAQ stock market index ('IXIC) range from 6 August 2015 to 1 September 2020. We divided the time period into two parts: pre-COVID (6 August 2015-31 January 2020) and COVID era (1 February 2020-1 September 2020). In each data set of crypto market and stock market indices, we have open price, intraday high price, intraday low price, close price (adjusted for splits), adjusted close price (adjusted for both dividends and splits) and volume. To better illustrate the relationship between crypto market data and stock market indexes, we calculate the daily log return using adjusted close price:

$$
\text { Return }_{t}=100 *\left[\ln \left(P_{t}\right)-\ln \left(P_{t-1}\right)\right] \text {, }
$$

where $P_{t}$ denotes the adjusted close price in USD at a time $t$.

We now document main statistical properties of time series for the returns of S\&P500 stock market index, NASDAQ stock market index, Bitcoin and Ethereum. Figure 1 illustrates the time evolution of prices, volumes and daily log-returns for S\&P500, NASDAQ, Bitcoin and Ethereum. We notice that both Bitcoin and Ethereum arrive their period specific highest price in December 2017 within our analysis period. After this period price peak, the crypro price dropped dramatically. The descriptive statistics of daily log-returns are reported in Table 1 . The daily returns of crypto markets exhibit high variability and excess kurtosis, both during pre-COVID and COVID era periods. The deviations from the Normal distribution are confirmed by the Jarque-Bera test that rejects the null hypothesis of normality.

We applied the Augmented Dicker-Fuller (ADF) unit-root test, which suggests stationarity of the log-returns. An ADF test tests the null hypothesis that a unit root is present in a time series sample. The alternative hypothesis is different depending on which version of the test is used, but is usually stationary or trend-stationary. In our case, we use the alternative hypothesis of stationary. This shows that the null hypothesis is rejected, and the time series of returns in each markets is stationary. These observations suggest that the crypto market is not as efficient as stock or foreign exchange markets, which display a complete lack of predictability (Lahmiri and Bekiros 2018).

Since early 2020, the COVID-19 wreaked unprecedented havoc on the world economies. Educational institutions, travel industry to public events, almost everything is either postponed or in limbo, which is inevitably going to affect businesses at every turn. Thousands of cases and deaths have already been recorded globally, and with the uncertainty on development of vaccines, the stock markets began to take many hits in terms of new lows. The S\&P 500 index hit a period low since 2008 when the world plunged into a financial crisis. The cryptocurrency market has even become more volatile and has also experienced one of the worst sudden declines. We also noticed from Figure 1 that both cryptos and stock market indexes became more uncertain since the COVID-19 outbreak in early 2020. The return prices and volumes of Bitcoin and Ethereum also surged since early 2020. 
Table 1. Descriptive statistics.

\begin{tabular}{lcccccccc}
\hline & \multicolumn{3}{c}{ Pre-COVID (Aug. 2015-Jan. 2020) } & \multicolumn{3}{c}{ COVID Era (Feb. 2020.-Sep. 2020 } \\
\hline Daily Log-Return & S\&P500 & Nasdaq & Bitcoin & Ethereum & S\&P500 & Nasdaq & Bitcoin & Ethereum \\
\hline Observations & 1129 & 1129 & 1640 & 1639 & 147 & 147 & 213 & 213 \\
Mean & 0.04 & 0.05 & 0.21 & 0.25 & 0.05 & 0.16 & 0.11 & 0.45 \\
Standard deviation & 0.86 & 1.04 & 3.89 & 7.09 & 2.72 & 2.71 & 4.61 & 5.92 \\
Skewness & -0.57 & -0.51 & -0.18 & -3.44 & -0.73 & -0.92 & -4.49 & -3.68 \\
Kurtosis & 4.12 & 3.15 & 4.72 & 72.46 & 5.13 & 5.27 & 48.02 & 35.51 \\
Augmented Dickey-Fuller (ADF) & $-10.98^{* *}$ & $-11.26^{* *}$ & $-10.93^{* *}$ & $-10.93^{* *}$ & $-5.64^{* *}$ & $-5.48^{* *}$ & $-5.16^{* *}$ & $-4.98^{* *}$ \\
Jarque-Bera & $862.50^{* * *}$ & $518.27^{* * *}$ & $1538.80^{* * *}$ & $362,486^{* * *}$ & $180.51^{* * *}$ & $197.22^{* * *}$ & $21,507^{* * *}$ & $11,855^{* * *}$ \\
\hline
\end{tabular}

Note: Entries marked with ${ }^{* * *}$ have empirical $p$-values $<0.01$ and ${ }^{* *} 0.01 \leq p<0.05$ under the null of non-stationary data for ADF test and the null of normally distributed data for Jarque-Bera test.

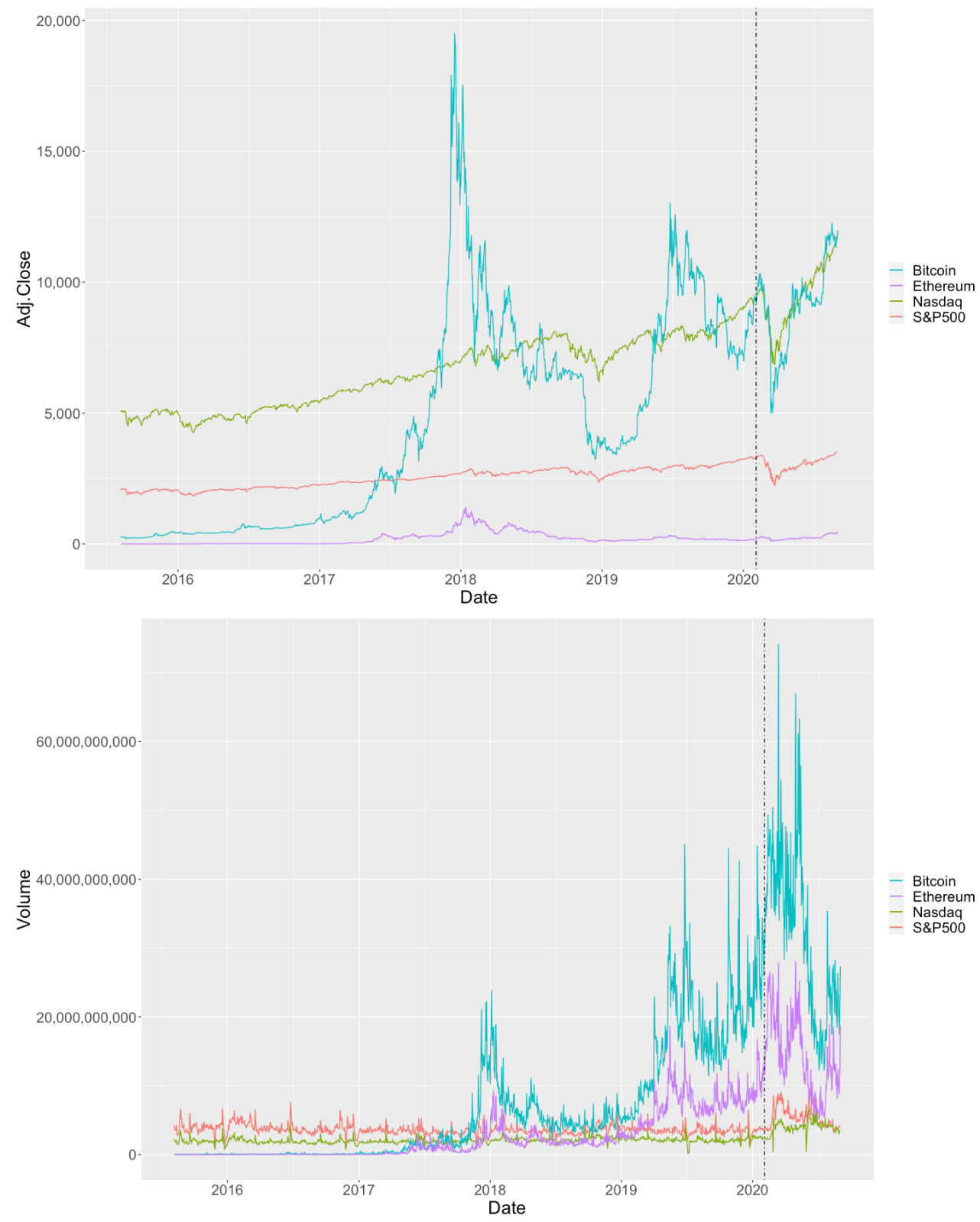

Figure 1. Cont. 

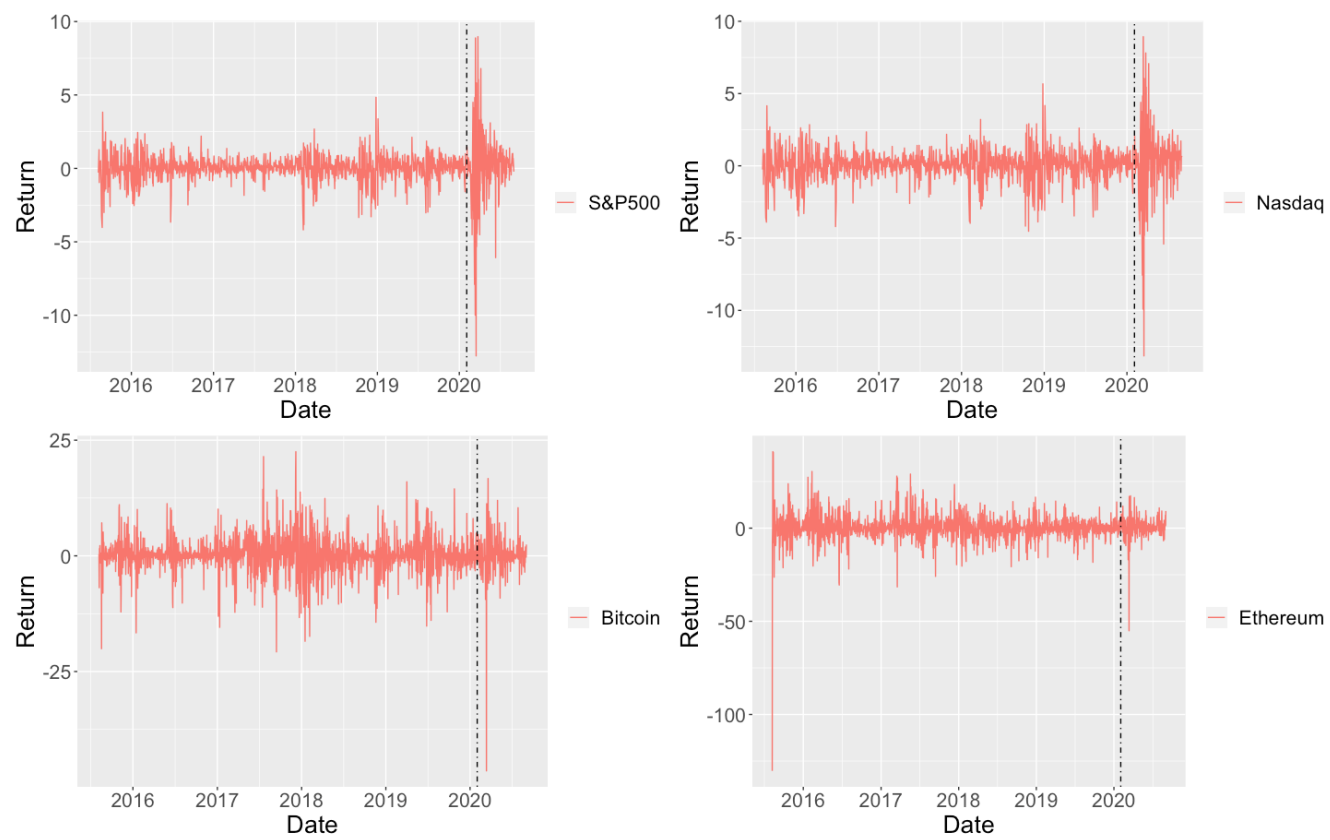

Figure 1. Plot of price, volume and daily log-returns.

\section{Entropy Profiles Method}

\subsection{Brief Introduction to Information Theory and Entropy}

Consider two variables $X$ and $Y$. Correlation between them may be ill defined when they are discrete, and may be a poor measure of "relation" when nonlinearity and/or non-Gaussianity is involved.

Let $\Re=\left\{a_{1}, a_{2}, \ldots, a_{M}\right\}$ be a finite set and $p$ be a proper probability mass function (PDF) on $\Re$. The amount of information needed to fully characterize all of the elements of this set consisting of $M$ discrete elements is defined by $I\left(\Re_{M}\right)=\log _{2} M$ and is known as Hartley's formula. Shannon (1948) built on Hartley's formula in the context of digitization and communications, to develop Shannon's entropy:

$$
H(p)=-\sum_{i=1}^{M} p_{i} \log \left(p_{i}\right)
$$

with $x \log (x)$ tending to zero as $x$ tends to zero. This information criterion measures the uncertainty or informational content that is implied by $p$. The entropy-uncertainty measure $H(p)$ reaches a maximum when $p_{1}=p_{2}=\ldots=p_{M}=1 / M$ (and is equal to Hartley's formula) and a minimum with a point mass function. It is emphasized here that $H(p)$ is a function of the probability distribution. For example, if $\eta$ is a random variable with possible distinct realizations $x_{1}, x_{2}, \ldots, x_{M}$ with probabilities $p_{1}, p_{2}, \ldots, p_{M}$, the entropy $H(p)$ does not depend on the values $x_{1}, x_{2}, \ldots, x_{M}$ of $\eta$. If, on the other hand, $\eta$ is a continuous random variable, then the entropy of a continuous density is

$$
H(x)=-\int p(x) \log (p(x)) d x,
$$

a differential entropy.

Renyi (1961) showed that, for a (sufficiently often) repeated experiment, one needs on average the amount $H(p)+\epsilon$ of zero-one symbols (for any positive $\epsilon$ ) in order to characterize an outcome of that experiment. Thus, it seems logical to "expect" that the outcome of an experiment contains $H(p)$ information.

Similarly, $H(p)$ is a measure of uncertainty about a specific possible outcome before observing it, which is equivalent to the amount of randomness represented by $p$. It is proportional to "variance" in the case of a Normal distribution. Thus, entropy is a far superior 
and robust measure of volatility/risk than variance for non Gaussian phenomena. It is indeed unique for any distribution, much as the characteristic function is, both representing all the moments of a distribution, which could be merely the mean and variance in the case of a Normal variable. Asset returns are not Gaussian!

Given a prior or competing distribution $q$, defined on $\Re$, the cross-entropy (Kullback and Leibler 1951) measure is

$$
I(p ; q)=\sum_{i=1}^{M} p_{i} \log \left(p_{i} / q_{i}\right)
$$

where a uniform $q$ reduces $I(p ; q)$ to $H(p)$. This measure reflects the gain in information with respect to $\Re$ resulting from the additional knowledge in $p$ relative to $q$. Like with $H(p), I(p ; q)$ is an information theoretic distance of $p$ from $q$. It can be symmetrized by averaging $I(p ; q)$ and $I(q ; p)$.

Facing the fundamental question of drawing inferences from limited and insufficient data, Jaynes proposed the maximum entropy (ME) principle, which he viewed as a generalization of Bernoulli and Laplace's Principle of Insufficient Reason.

Given $T$ constraints, perhaps in the form of moments, Jaynes proposed the ME method, which is to maximize $H(p)$ subject to the $T$ structural constraints. Thus, given moment conditions, $X_{t}(t=1,2, \ldots, T)$, where $T<M$, the ME principle prescribes choosing the $p\left(a_{i}\right)$ that maximizes $H(p)$ subject to the given constraints (moments) of the problem. The solution to this underdetermined problem is

$$
\widehat{p\left(a_{i}\right)} \propto \exp \left\{-\sum_{t} \hat{\lambda}_{t} X_{t}\left(a_{i}\right)\right\}
$$

where $\lambda$ are the $T$ Lagrange multipliers, and $\hat{\lambda}$ are the values of the optimal solution (estimated values) of $\lambda$. Naturally, if no constraints are imposed, $H(p)$ reaches its maximum value and the $p$ are distributed uniformly.

Building on Shannon's work, a number of generalized entropies and information measures were developed. Starting with the idea of describing the gain of information, (Renyi 1970) developed the entropy of order $\alpha$ for incomplete random variables. The relevant generalized entropy measure of a proper probability distribution is

$$
H_{\alpha}^{R}(p)=\frac{1}{1-\alpha} \log \sum_{k} p_{k}^{\alpha}
$$

Shannon measure is a special case of this measure where $\alpha \rightarrow 1$. Similarly, the Renyi cross-entropy of order $\alpha$ is

$$
I_{\alpha}^{R}(x \mid y)=I_{\alpha}^{R}(p, q)=\frac{1}{1-\alpha} \log \sum_{k} \frac{p_{k}^{\alpha}}{q_{k}^{\alpha-1}},
$$

which is equal to the traditional cross-entropy measure as $\alpha \rightarrow 1$. Only one member of these "divergence" measures is a metric, which we define below.

Entropy has been actively considered in finance theory since at least 1999. According to (Gulko 1999), "entropy pricing theory" suggests that in information efficient markets, perfectly uncertain market beliefs must prevail. Using entropy to measure market uncertainty, entropy-maximizing market beliefs must prevail. One can derive (entropy) optimal asset pricing models that are similar to the Black-Scholes model (with the log-normal distribution replaced by other probability distributions).

\subsection{Using Entropy to Test Equality of Univariate Densities}

Maasoumi and Racine (2002) considered a metric entropy that is useful for testing for equality of densities for two univariate random variables $X$ and $Y$. The function 
computes the nonparametric metric entropy (normalized Hellinger, or (Granger et al. 2004) for testing the null of equality of two univariate density (or probability) functions. For continuous variables,

$$
\begin{aligned}
S_{\rho} & =\frac{1}{2} \int\left(f_{1}^{1 / 2}-f_{2}^{1 / 2}\right)^{2} d x \\
& =\frac{1}{2} \int\left(1-\frac{f_{2}^{1 / 2}}{f_{1}^{1 / 2}}\right)^{2} d F_{1}(x),
\end{aligned}
$$

where $f_{1}=f(x)$ and $f_{2}=f(y)$ are the marginal densities of the random variables $X$ and $Y$. The second expression is in a moment from which is often replaced with a sample average, especially for theoritical developments. If the density of $X$ and the density of $Y$ are equal, this metric will yield the value zero, and is otherwise positive and less than one. We use $S_{\rho}$ to test the distance between crypto density and stock market index density. Some properties this entropy measure $S_{\rho}$ are given in (Granger et al. 2000) and (Giannerini et al. 2015). In particular, the modulus of $S_{\rho}$ is between 0 and unity; $S_{\rho}$ is equal to or has a simple relationship with the (linear) correlation coefficient in the case of a bivariate normal distribution; $S_{\rho}$ is metric, that is, it is a true measure of distance and not just of "divergence". This is especially important in our applications where triangularity property is required in meaningful comparative assessments of several distances and asset classes.

Software for nonparametric kernel smoothing implementation of this metric is made available in R (NP package) among others. For the kernel function, we employ the widely used nonparametric second-order Gaussian kernel, while bandwidths are selected via likelihood cross-validation (Nakayama and Silverman 1986). Bootstrap is conducted via resampling with replacement from the pooled empirical distributions of $X$ and $Y$ under the null hypothesis of equality.

We estimate the metric $S_{\rho}$ for the daily returns data for $x=$ Return $_{\text {crypto }}$ and $y=$ Return $_{\text {stock }}$. Table 2 shows the $S_{\rho}$ values and the corresponding $p$-values. As was noted in (Granger et al. 2000) and (Skaug and Tjostheim 1996), the asymptotic distribution of $S_{\rho}$ is unreliable for practical inference. We therefore compute $p$-values by resampling the statistic under the null of equality.

Examining Table 2, we see that $S_{\rho}$ is smallest between $x=$ Bitcoin and $y=$ NASDAQ, both during the pre-COVID and COVID era periods, which indicates that the distance between the densities of Bitcoin daily returns and NASDAQ daily returns is smaller than other combinations. The p-value shows that the result is significant. By visualizing the result in Figures 2-5, we can also see the Bitcoin daily returns density and the NASDAQ stock market index daily returns density have similar shapes. While during COVID era, also S\&P500 returns distribution is statistically closely dependent on, and indifferent from Bitcoin's.

Comparing $S_{\rho}$ before and after the COVID-19 outbreak, we conclude that the values of $S_{\rho}$ decrease generally in all cases, sometimes dramatically. This suggests that the densities of crypto and stock index returns became more similar with the advent of COVID-19. This mostly due to a large change in the distribution of major stock indices, but also party due to a smaller movement in crypto distributions.

Table 3 reveals the entropy metric $S_{\rho}$ of the assets themselves pre-COVID and COVID era. By doing so, we can see if the difference between the cryptos and stocks is partly due to specific asset change caused by the effect of COVID-19. The results show that the distributions of S\&P500 and NASDAQ changed dramatically and significantly before and after the COVID-19 outbreak, which indicates that the changes of $S_{\rho}$ between cryptos and stocks may mainly caused by the changes of stocks' distributions. We will dive deeper on this part in Section 4. 
Table 2. Test equality of univariate densities: cryptos and stocks.

\begin{tabular}{lccccc}
\hline & Pre-COVID (Aug. 2015-Jan. 2020) & \multicolumn{2}{c}{ COVID Era (Feb. 2020-Sep. 2020) } & Difference \\
\hline Daily Log-Return & S_rho & $p$-Value & S_rho & $p$-Value & -0.16 \\
\hline S\&P500 and Bitcoin & 0.20 & $2.22 \times 10^{-16 * * *}$ & 0.04 & 0.1010 & -0.25 \\
S\&P500 and Ethereum & 0.33 & $2.22 \times 10^{-16 * * *}$ & 0.08 & $2.22 \times 10^{-16 * *}$ & -0.12 \\
NASDAQ and Bitcoin & 0.16 & $2.22 \times 10^{-16 * * *}$ & 0.04 & $0.0404^{*}$ & -0.20 \\
NASDAQ and Ethereum & 0.28 & $2.22 \times 10^{-16 * * *}$ & 0.08 & $2.22 \times 10^{-16 * *}$ & -
\end{tabular}

Note: Entries marked with ${ }^{* *}$ have empirical $p$-values $<0.01$ and ${ }^{*} 0.05 \leq p<0.10$ under the null of independence of returns.

Table 3. Test equality of univariate densities: assets with themselves pre-COVID and COVID era.

\begin{tabular}{lcc}
\hline Daily Log-Return & S_rho & $p$-Value \\
\hline S\&P500 with itself pre-COVID and COVID era & 0.13 & $<2.22 \times 10^{-16 * * *}$ \\
NASDAQ with itself pre-COVID and COVID era & 0.10 & $<2.22 \times 10^{-16 * * *}$ \\
Bitcoin with itself pre-COVID and COVID era & 0.02 & 0.3737 \\
Ethereum with itself pre-COVID and COVID era & 0.02 & $0.0303^{*}$
\end{tabular}

Note: Entries marked with ${ }^{* *}$ have empirical $p$-values $<0.01$ and ${ }^{*} 0.05 \leq p<0.10$ under the null of independence of returns.
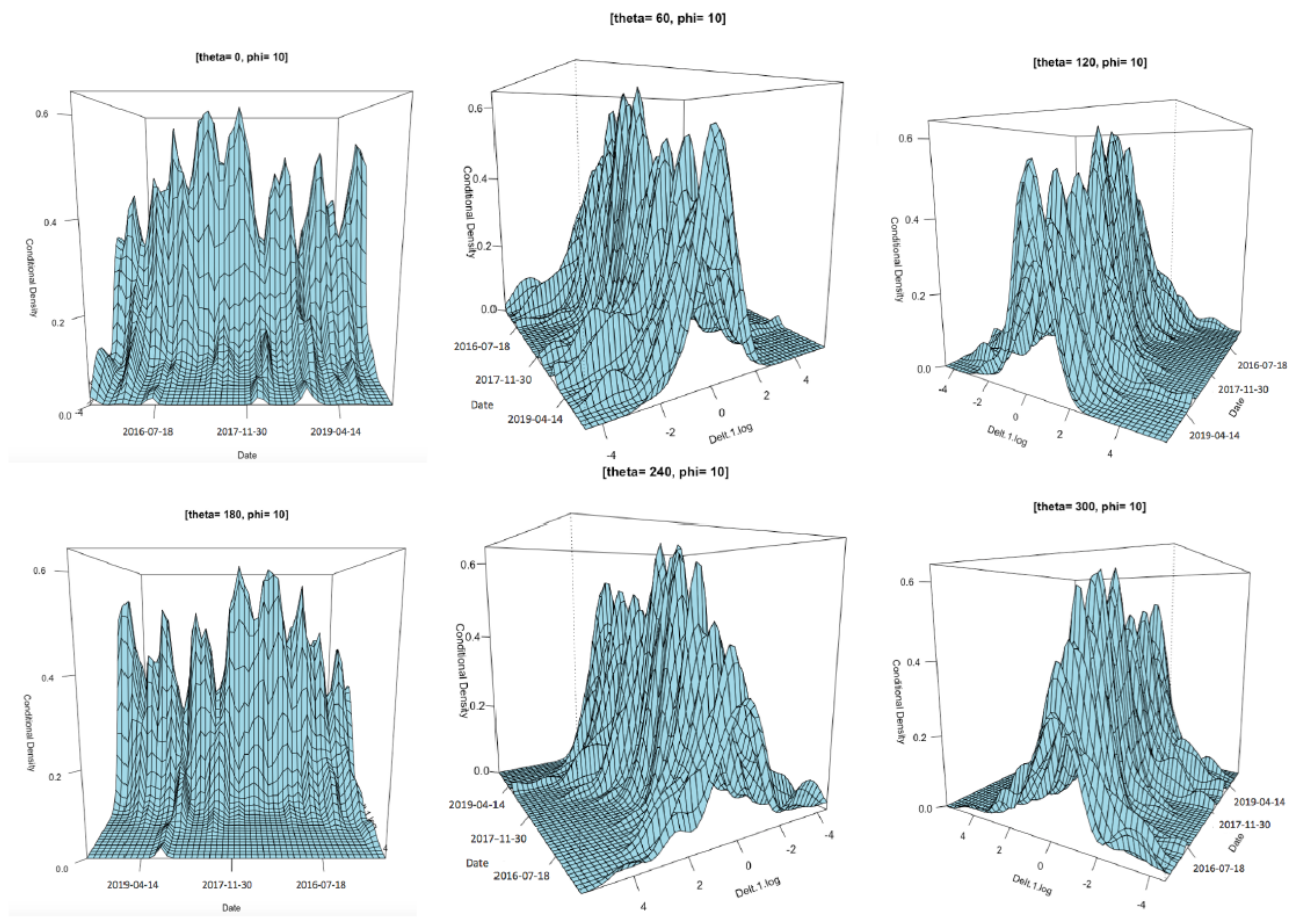

Figure 2. Density of NASDAQ: pre-COVID. 

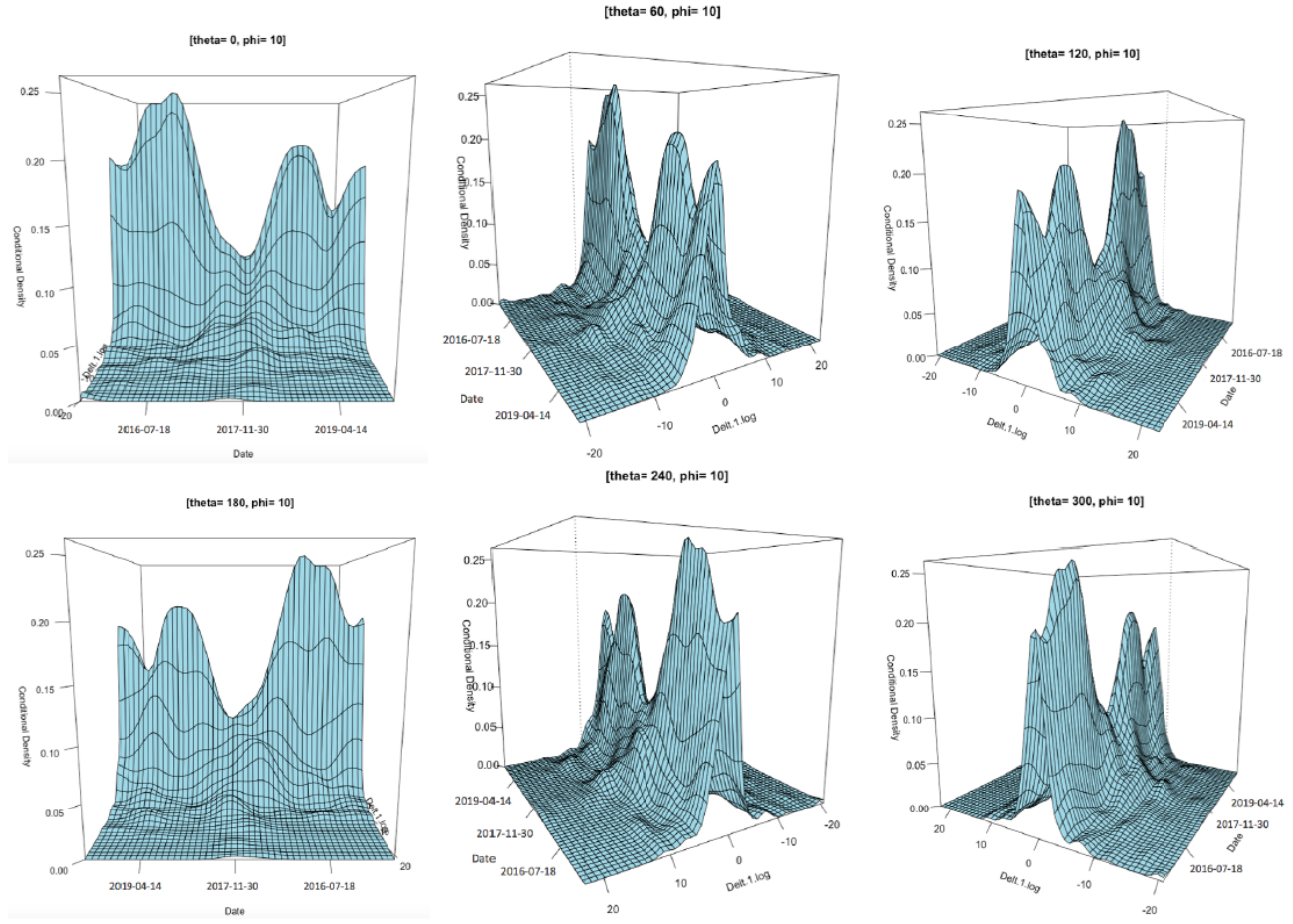

Figure 3. Density of Bitcoin: pre-COVID.
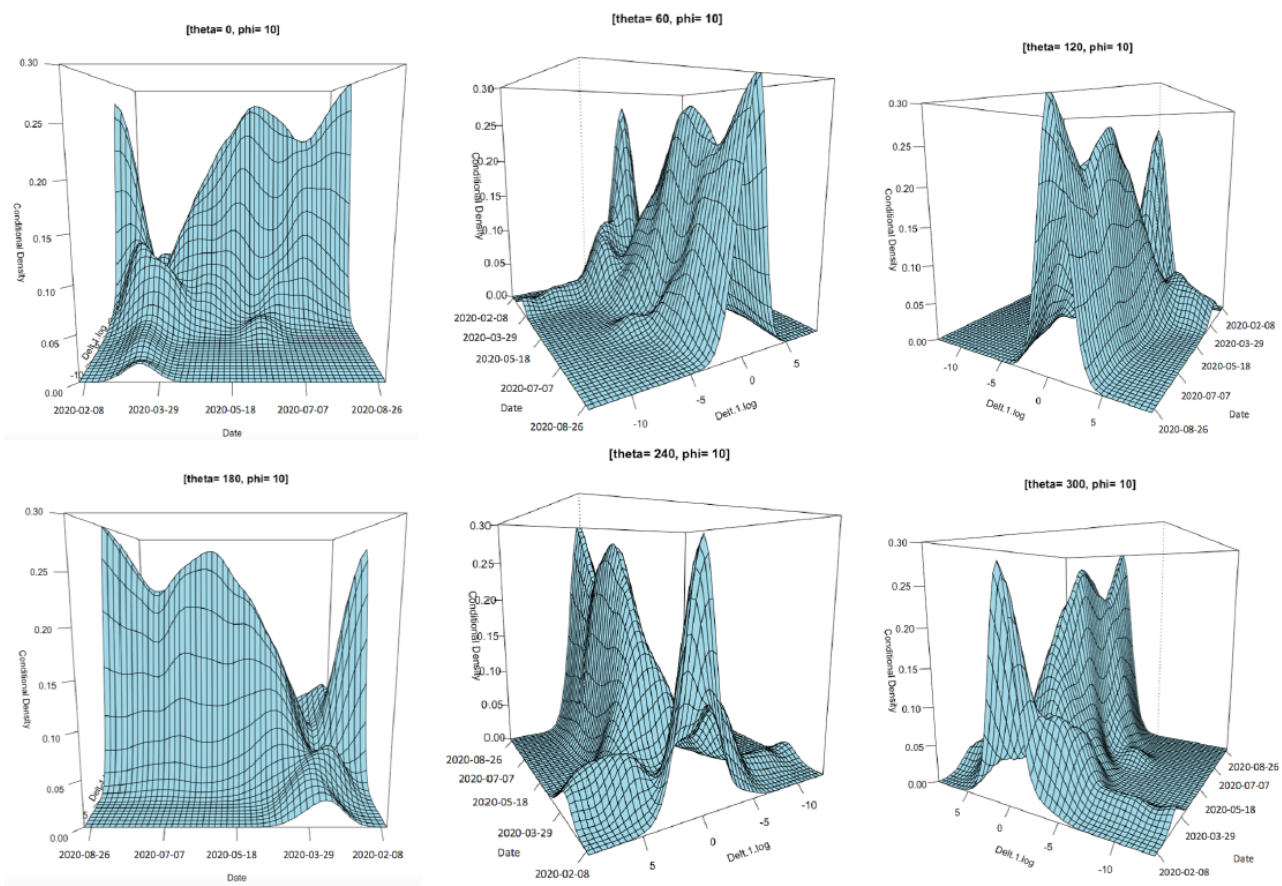

Figure 4. Density of NASDAQ: COVID era. 

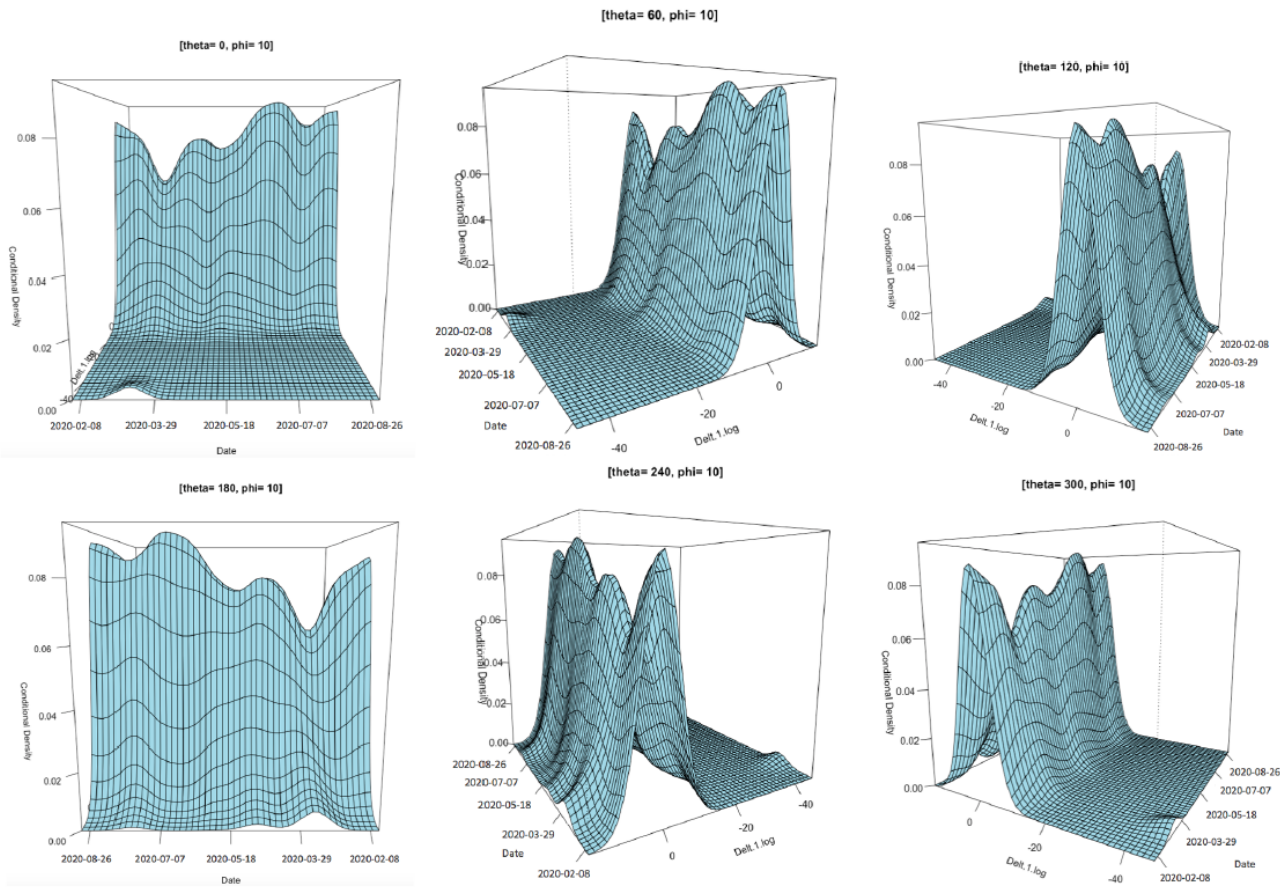

Figure 5. Density of Bitcoin: COVID era.

\subsection{Similarity with Select Asset Classes}

In this part, we apply the same method to test the equality of densities for daily returns of Bitcoin and stocks in different industry groups. The data for daily stock returns in different industries comes from Kenneth R. French 30 Industry Portfolios ${ }^{2}$. We use the daily average value weighted returns for 30 industry portfolios data. The 30 industry portfolios include: Food Products (Food), Beer and Liquor (Beer), Tobacco Products (Smoke), Recreation (Games), Printing and Publishing (Books), Consumer Goods (Hshld), Apparel (Clths), Healthcare (Hlth), Medical Equipment, Pharmaceutical Products, Chemicals (Chems), Textiles (Txtls), Construction and Construction Materials (Cnstr), Steel Works Etc (Steel), Fabricated Products and Machinery (Fabpr), Electrical Equipment (Elceq), Automobiles and Trucks (Autos), Aircraft, ships, and railroad equipment (Carry), Precious Metals, Non-Metallic, and Industrial Metal Mining (Mines), Coal (Coal), Petroleum and Natural Gas (Oil), Utilities (Util), Communication (Telcm), Personal and Business Services (Servs), Business Equipment (Buseq), Business Supplies and Shipping Containers (Paper), Transportation (Trans), Wholesale (Whlsl), Retail (Rtail), Restaraunts, Hotels, Motels (Meals), Banking, Insurance, Real Estate, Trading (Fin), Everything Else (Other). We apply the nonparametric entropy metrics test of equality of densities proposed in (Renyi 1961), described above, where $f_{1}=f(x)$ and $f_{2}=f(y)$ are the marginal densities of daily returns of Bitcoin and stocks in different industries, respectively.

From Table 4, we calculated the entropy measures between Bitcoin and select asset classes. During pre-COVID period, the density of Bitcoin daily return has smallest distance with the density of Coal industry daily return. The $S_{\rho}$ between these two densities is 0.02 and statistically significant. The density of Bitcoin daily return also has small distances with densities of Steel Works Etc, as well as Precious Metals, Non-Metallic, and Industrial Metal Mining industries, with $S_{\rho}$ values of 0.07 and 0.09, respectively. During COVID era, the density of Bitcoin daily return has smallest distance with the density of Business Supplies and Shipping Containers, Utilities, Tobacco Products and Restaraunts, Hotels, Motels industries daily returns, with $S_{\rho}$ values of 0.03 . Comparing $S_{\rho}$ before and after the COVID-19 outbreak, we conclude that the values of $S_{\rho}$ decrease generally in all cases. This is consistent with our findings with stock indexes in the previous section, which indicates that forecasting cryptos' performance could be more feasible during COVID era. 
We also calculated the $S_{\rho}$ with select asset classes with themselves before and after the COVID-19 outbreak (see column 2 in Table 4). It is clear that for all industry groups during COVID era, the asset distributions diverge from their own pre-COVID distributions, and the distribution divergence of industry groups are more significant comparing with cryptos' (shown in Table 3).

Table 4. Entropy measure between Bitcoin and different Industries.

\begin{tabular}{|c|c|c|c|c|c|c|c|}
\hline \multirow[b]{2}{*}{ Daily Log-Return } & \multicolumn{2}{|c|}{ Pre-COVID and COVID Era with Itself } & \multicolumn{2}{|c|}{ Pre-COVID with Bitcoin } & \multicolumn{2}{|c|}{ COVID Era with Bitcoin } & \multirow[t]{2}{*}{ Difference } \\
\hline & S_rho & $p$-Value & S_rho & $p$-Value & S_rho & $p$-Value & \\
\hline Food & 0.16 & $<2.22 \times 10^{-16 * * *}$ & 0.22 & $<2.22 \times 10^{-16 * * *}$ & 0.04 & 0.0808 & -0.18 \\
\hline Beer & 0.14 & $<2.22 \times 10^{-16 * * *}$ & 0.21 & $<2.22 \times 10^{-16 * * *}$ & 0.07 & 0.1010 & -0.14 \\
\hline Smoke & 0.14 & $<2.22 \times 10^{-16 * * *}$ & 0.14 & $<2.22 \times 10^{-16 * * *}$ & 0.03 & 0.2121 & -0.11 \\
\hline Games & 0.09 & $<2.22 \times 10^{-16 * * *}$ & 0.10 & $<2.22 \times 10^{-16 * * *}$ & 0.05 & $0.0202 *$ & -0.05 \\
\hline Books & 0.19 & $<2.22 \times 10^{-16 * * *}$ & 0.15 & $<2.22 \times 10^{-16 * * *}$ & 0.04 & 0.0909 & -0.11 \\
\hline Hshld & 0.14 & $<2.22 \times 10^{-16 * * *}$ & 0.21 & $<2.22 \times 10^{-16 * * *}$ & 0.04 & 0.4040 & -0.17 \\
\hline Clths & 0.20 & $<2.22 \times 10^{-16 * * *}$ & 0.12 & $<2.22 \times 10^{-16 * * *}$ & 0.04 & 0.1212 & -0.08 \\
\hline Hlth & 0.12 & $<2.22 \times 10^{-16 * * *}$ & 0.17 & $<2.22 \times 10^{-16 * * *}$ & 0.04 & 0.1717 & -0.13 \\
\hline Chems & 0.21 & $<2.22 \times 10^{-16 * * *}$ & 0.15 & $<2.22 \times 10^{-16 * * *}$ & 0.04 & 0.1414 & -0.11 \\
\hline Txtls & 0.26 & $<2.22 \times 10^{-16 * * *}$ & 0.11 & $<2.22 \times 10^{-16 * * *}$ & 0.07 & $0.0101 *$ & -0.04 \\
\hline Cnstr & 0.23 & $<2.22 \times 10^{-16 * * *}$ & 0.14 & $<2.22 \times 10^{-16 * * *}$ & 0.04 & 0.2020 & -0.10 \\
\hline Steel & 0.14 & $<2.22 \times 10^{-16 * * *}$ & 0.07 & $<2.22 \times 10^{-16 * * *}$ & 0.05 & $0.0202 *$ & -0.02 \\
\hline Fabpr & 0.19 & $<2.22 \times 10^{-16 * * *}$ & 0.13 & $<2.22 \times 10^{-16 * * *}$ & 0.04 & 0.0808 & -0.09 \\
\hline Elceq & 0.22 & $<2.22 \times 10^{-16 * * *}$ & 0.14 & $<2.22 \times 10^{-16 * * *}$ & 0.04 & 0.1111 & -0.10 \\
\hline Autos & 0.21 & $<2.22 \times 10^{-16 * * *}$ & 0.12 & $<2.22 \times 10^{-16 * * *}$ & 0.04 & 0.1212 & -0.08 \\
\hline Carry & 0.27 & $<2.22 \times 10^{-16 * * *}$ & 0.15 & $<2.22 \times 10^{-16 * * *}$ & 0.06 & $0.0202 *$ & -0.08 \\
\hline Mines & 0.09 & $<2.22 \times 10^{-16 * * *}$ & 0.09 & $<2.22 \times 10^{-16 * * *}$ & 0.05 & 0.0505 & -0.05 \\
\hline Coal & 0.09 & $<2.22 \times 10^{-16 * * *}$ & 0.02 & $<2.22 \times 10^{-16 * * *}$ & 0.09 & $<2.22 \times 10^{-16 * * *}$ & 0.07 \\
\hline Oil & 0.22 & $<2.22 \times 10^{-16 * * *}$ & 0.11 & $<2.22 \times 10^{-16 * * *}$ & 0.05 & $0.0101 *$ & -0.05 \\
\hline Util & 0.22 & $<2.22 \times 10^{-16 * * *}$ & 0.22 & $<2.22 \times 10^{-16 * * *}$ & 0.03 & 0.3939 & -0.18 \\
\hline Telcm & 0.19 & $<2.22 \times 10^{-16 * * *}$ & 0.20 & $<2.22 \times 10^{-16 * * *}$ & 0.04 & 0.1313 & -0.16 \\
\hline Servs & 0.14 & $<2.22 \times 10^{-16 * * *}$ & 0.16 & $<2.22 \times 10^{-16 * * *}$ & 0.05 & 0.1111 & -0.11 \\
\hline Buseq & 0.13 & $<2.22 \times 10^{-16 * * *}$ & 0.14 & $<2.22 \times 10^{-16 * * *}$ & 0.04 & 0.1717 & -0.10 \\
\hline Paper & 0.17 & $<2.22 \times 10^{-16 * * *}$ & 0.18 & $<2.22 \times 10^{-16 * * *}$ & 0.03 & 0.3535 & -0.15 \\
\hline Trans & 0.18 & $<2.22 \times 10^{-16 * * *}$ & 0.15 & $<2.22 \times 10^{-16 * * *}$ & 0.04 & 0.1515 & -0.11 \\
\hline Whlsl & 0.24 & $<2.22 \times 10^{-16 * * *}$ & 0.19 & $<2.22 \times 10^{-16 * * *}$ & 0.04 & 0.2020 & -0.15 \\
\hline Rtail & 0.10 & $<2.22 \times 10^{-16 * * *}$ & 0.18 & $<2.22 \times 10^{-16 * * *}$ & 0.08 & $<2.22 \times 10^{-16 * * *}$ & -0.10 \\
\hline Meals & 0.24 & $<2.22 \times 10^{-16 * * *}$ & 0.20 & $<2.22 \times 10^{-16 * * *}$ & 0.03 & 0.2626 & -0.17 \\
\hline Fin & 0.25 & $<2.22 \times 10^{-16 * * *}$ & 0.16 & $<2.22 \times 10^{-16 * * *}$ & 0.05 & 0.1010 & -0.11 \\
\hline Other & 0.20 & $<2.22 \times 10^{-16 * * *}$ & 0.20 & $<2.22 \times 10^{-16 * * *}$ & 0.04 & 0.1010 & -0.16 \\
\hline
\end{tabular}

Note: Entries marked with ${ }^{* *}$ have empirical $p$-values $<0.01$ and ${ }^{*} 0.05 \leq p<0.10$ under the null of independence of returns.

\subsection{Testing General Nonlinear Co-Dependence}

The above test of (Renyi 1961) may be employed for testing stochastic independence of any two random variables $X$ and $Y$. Let $f_{1}=f\left(x_{i}, y_{i}\right)$ be the joint density and $f_{2}=g\left(x_{i}\right) * h\left(y_{i}\right)$ be the product of the marginal densities. The unknown density functions are replaced with nonparametric kernel estimates. The methodology is as before, with the null of independence imposed in the bootstrap resampling implementation of the test. Bandwidths are obtained via likelihood cross-validation by default for the marginal and joint densities.

The results are in Table 5. There is significant dependence only between Bitcoin and NASDAQ before the COVID-19 outbreak. During COVID era, independence is comfortably rejected for all pairings. The two situations represent very radical changes in the status of cryptos for portfolio diversification. 
Table 5. Independence test.

\begin{tabular}{lcccc}
\hline & \multicolumn{2}{c}{ Pre-COVID (Aug. 2015-Jan. 2020) } & \multicolumn{2}{c}{ COVID Era (Feb. 2020-Sep. 2020) } \\
\hline Daily Log-Return & S_rho & $p$-Value & S_rho & $p$-Value \\
\hline S\&P500 and Bitcoin & 0.0085 & $0.0303 *$ & 0.0148 & $2.22 \times 10^{-16 * * *}$ \\
S\&P500 and Ethereum & 0.0076 & 0.5758 & 0.0172 & $2.22 \times 10^{-16 * * *}$ \\
NASDAQ and Bitcoin & 0.0072 & $0.0101 *$ & 0.0163 & $2.22 \times 10^{-16 * * *}$ \\
NASDAQ and Ethereum & 0.0061 & 0.6061 & 0.0178 & $2.22 \times 10^{-16 * * *}$ \\
\hline
\end{tabular}

Note: Entries marked with ${ }^{* *}$ have empirical $p$-values $<0.01$ and ${ }^{*} 0.05 \leq p<0.10$ under the null of independence of returns.

\section{Difference-in-Differences Analysis}

Difference in differences (Diff-in-diff) is a statistical technique used in econometrics and quantitative research that attempts to mimic an experimental research design using observational study data, by studying the differential effect of a treatment on a "treatment group" versus a "control group" in a natural experiment. It calculates the effect of a treatment on an outcome by comparing the average change over time in the outcome variable for the treatment group, compared to the average change over time for the control group (Card and Krueger 1993).

Before we construct our Diff-in-diff model, we would like to emphasize that the entropy metrics exhibit linear decomposition property. The reason why we can decompose $S_{\rho}$ is that it is a metric, which means it satisfies the triangularity property of distances. Therefore, we can write the entropy metric between stock and crypto during COVID era as the summation of the entropy metric between them during pre-COVID period plus a time trend $\lambda_{t}$ and plus the COVID effect.

$$
S_{\rho}\left(f_{s_{i}, t_{2}}, f_{c_{j}, t_{2}}\right)=S_{\rho}\left(f_{s_{i}, t_{1}}, f_{\mathcal{c}_{j}, t_{1}}\right)+\lambda_{t}+\operatorname{COVID}+\epsilon_{i, j},
$$

where $S_{\rho}\left(f_{s_{i}, t_{2}}, f_{c_{j}, t_{2}}\right)$ stands for the entropy metric between stock $i$ and crypto $j$ during COVID era, and $S_{\rho}\left(f_{s_{i}, t_{1}}, f_{c_{j}, t_{1}}\right)$ stands for the entropy metric between stock $i$ and crypto $j$ during pre-COVID period. $\lambda_{t}$ is the time trend defined by $\lambda_{t}=S_{\rho}\left(f_{s_{i}, t_{2}}, f_{s_{i}, t_{1}}\right)+$ $S_{\rho}\left(f_{c_{i}, t_{2}}, f_{c_{j}, t_{1}}\right)$, which measures the entropy metric of both stock $i$ and crypto $j$ from preCOVID period to COVID era with itself. COVID is the effect of exogenous shock provided by COVID-19 to the entropy metrics. $\epsilon_{i, j}$ is the residual term.

Since we have already calculated the distribution distances between assets in the previous sections, from Equation (9), we can easily estimate the COVID effect on the entropy metrics, say $\widehat{C O V I D}$. Using entropy metrics $S_{\rho}$ between Bitcoin and other assets (including S\&P500, NASDAQ, the the 30 industry portfolios), we can estimate the COVID effect $\widehat{C O V I D}=-0.30$. This indicates that after the broke out of COVID-19 pandemic, the distributions of stocks and cryptos became more similar and less independent, quantitatively, the entropy metrics decrease by -0.30 in average.

Next, we follow Card and Krueger (1994) to construct our Diff-in-diff model:

$$
S_{\rho}\left(f_{A_{i}, t_{j}}, f_{0}\right)=\beta_{0}+\beta_{1} * \text { Covid }+\beta_{2} * \text { Crypto }+\beta^{D I D} *(\text { Covid } * \text { Crypto })+\epsilon,
$$

where the dependent variable $S_{\rho}\left(f_{A_{i}, t_{j}}, f_{0}\right)$ is our variable of interest, it stands for the entropy metric between asset $i$ 's distribution at time $j, f_{A_{i}, t_{j}}$, and a benchmark distribution $f_{0}$. Crypto and Covid are dummy variables. Crypto equals to 1 if the asset is a crypto, while it equals 0 if the asset is stock. Covid equals to 1 if during the COVID era and it equals to 0 if during the pre-COVID period. The coefficient for the interaction term, Covid $*$ Crypto, is the Diff-in-diff estimator. In this way, we construct our Diff-in-diff model for entropy metric.

We come up with a new method to use our nonparametric entropy metric to estimate the Diff-in-diff estimator. In Table 6, we show the decomposition of the Diff-in-diff analysis. The reason why we can decompose $S_{\rho}$ is that it is a metric, which means it satisfies 
the triangularity property of distances. If you take three points, A, B and C, the distance between any of those points is smaller than the total of the other two distances. Furthermore, note that $S_{\rho}$ is a "squared integral". The second line in Equation (8) also tells us that it is a simple expectation of $1-\left(f_{2} / f_{1}\right)^{1 / 2}$. This is equal to metric developed by Bhathacharya as a measure of relations between two variables. By algebra, we can derive the Diff-in-diff estimator as the entropy metrics between stocks and crytos during the COVID era subtract the entropy metric between them during pre-COVID period: $\hat{\beta}^{D I D}=S_{\rho}\left(f_{s_{i}, t_{2}}, f_{c_{j}, t_{2}}\right)-$ $S_{\rho}\left(f_{s_{i}, t_{1}}, f_{c_{j}, t_{1}}\right)$.

Table 6. DID decomposition.

\begin{tabular}{lccc}
\hline Distribution & Stock & Crypto & Difference \\
\hline pre-COVID & $S_{\rho}\left(f_{s_{i}, t_{1}}, f_{0}\right)$ & $S_{\rho}\left(f_{c_{j}, t_{1}}, f_{0}\right)$ & $S_{\rho}\left(f_{s_{i}, t_{1}}, f_{c_{j}, t_{1}}\right)$ \\
COVID era & $S_{\rho}\left(f_{s_{i}, t_{2}}, f_{0}\right)$ & $S_{\rho}\left(f_{c_{j}, t_{2}}, f_{0}\right)$ & $S_{\rho}\left(f_{s_{i}, t_{2}}, f_{c_{j}, t_{2}}\right)$ \\
\hline Change & $S_{\rho}\left(f_{s_{i}, t_{2}}, f_{s_{i}, t_{1}}\right)$ & $S_{\rho}\left(f_{c_{j}, t_{2}}, f_{c_{j}, t_{1}}\right)$ & $S_{\rho}\left(f_{s_{i}, t_{2}}, f_{c_{j}, t_{2}}\right)-S_{\rho}\left(f_{s_{i}, t_{1}}, f_{c_{j}, t_{1}}\right)$ \\
\hline
\end{tabular}

\section{Conclusions}

This paper investigates the similarity and co-dependence between cryptocurrencies, daily returns and stock daily returns, before and after the COVID-19 outbreak in early 2020.

Data exhibited different features before and after the COVID-19 outbreak. There is a similarity between the Bitcoin and NASDAQ stock market index with or without COVID19. The similarity and dependence between cryptos and stock market indexes has become stronger after the COVID-19 outbreak. Our findings are robust to model misspecification, and avoid linear measures of dependence and correlation. The entropy profiles method and time series models play different roles in forecasting the cryptocurrency return volatility, and these approaches are complimentary. The time series models elaborate the dynamic movement of returns, on average (conditional mean models). The entropy profiles method is a nonparametric approach which reveals the evolution of the entire distributions and their quantiles. In this paper, we have several findings: Firstly, we found that during the pre-COVID period, NASDAQ and Bitcoin return's distributions are the most similar. Secondly, we can see during the COVID era, the distances between all asset returns have declined by $75 \%$ or more, and most of these changes are caused by changes of stock return distributions. We also found that the asset group with the closest similarity with Bitcoin are Coal, Steel and Mining industries during pre-COVID period, and Business Supplies, Utilities, Tobacco Products and Restaurants, Hotels, Motels industries, compared to several others during COVID era. Finally, through non-linear co-dependence test, we found that during the COVID era, the densities of stocks and cryptos became more similar and less independent. These results are meaningful because we revealed the similarity and dependence structure between crypto and stock distributions. This can be useful in applying existing theories on stocks to cryptos.

As for future directions of this study, we plan to examine newer data as we have observe the effective vaccines rollout, stock market volatility and the crypto prices peak to a new high in 2021. We believe that the examination of newer data will drive more promising and effective policy implications.

Author Contributions: Conceptualization, E.M. and X.W.; methodology, E.M. and X.W.; software, X.W.; validation, E.M. and X.W.; formal analysis, E.M. and X.W.; investigation, E.M. and X.W.; resources, E.M. and X.W.; data curation, E.M. and X.W.; writing-original draft preparation, X.W.; writing-review and editing, E.M.; visualization, X.W.; supervision, E.M.; project administration, E.M.; funding acquisition, N/A. All authors have read and agreed to the published version of the manuscript.

Funding: This research received no external funding. 
Data Availability Statement: Publicly available datasets were analyzed in this study. This data can be found here: https:/ / finance.yahoo.com (accessed on 1 September 2020) and http://mba. tuck.dartmouth.edu/pages / faculty/ken.french/Data_Library/det_30_ind_port.html (accessed on 1 September 2020).

Conflicts of Interest: The authors declare no conflict of interest.

\section{Notes}

1 https:// finance.yahoo.com (accessed on 1 September 2020)

2 http://mba.tuck.dartmouth.edu/pages/faculty/ken.french/Data_Library/det_30_ind_port.html (accessed on 1 September 2020)

\section{References}

Al-Khazali, Osamah, Bouri Elie, and David Roubaud. 2018. The impact of positive and negative macroeconomic news surprises: Gold versus Bitcoin. Economics Bulletin 38: 373-82.

Balcilar, Mehmet, Elie Bouri, Rangan Gupta, and David Roubaud. 2017. Can volume predict Bitcoin returns and volatility? A quantiles-based approach. Economic Modelling 64: 74-81. [CrossRef]

Balcombe, Kelvin, and Iain Fraser. 2017. Do bubbles have an explosive signature in markov switching models? Economic Modelling 66: 81-100. [CrossRef]

Bariviera, Aurelio F. 2017. The inefficiency of Bitcoin revisited: A dynamic approach. Economics Letters 161: 1-4. [CrossRef]

Bariviera, Aurelio F., Basgall María José, Hasperué Waldo, and Marcelo Naiouf. 2017. Some stylized facts of the Bitcoin market. Physica A: Statistical Mechanics and its Applications 484: 82-90. [CrossRef]

Baur, Dirk G., and Thomas Dimpfl. 2018. Asymmetric volatility in cryptocurrencies. Economics Letters 173: 148-51. [CrossRef]

Begusic, Stjepan, Zvonko Kostanjčar, H. Eugene Stanley, and Boris Podobnik. 2018. Scaling properties of extreme price fluctuations in Bitcoin markets. Physica A: Statistical Mechanics and its Applications 510: 400-06. [CrossRef]

Bouri, Elie, Peter Molnár, Georges Azzi, David Roubaud, and Lars Ivar Hagfors. 2017. On the hedge and safe haven properties of Bitcoin: Is it really more than a diversifier? Finance Research Letters 20: 192-98. [CrossRef]

Card, David, and Alan B. Krueger. 1993. Minimum wages and employment: A case study of the fast-food industry in New Jersey and Pennsylvania: Reply. American Economic Review 90: 1397-420. [CrossRef]

Dyhrberg, Anne Haubo. 2016. Bitcoin, gold and the dollar-A GARCH volatility analysis. Finance Research Letters 16: 85-92. [CrossRef]

Giannerini, Simone, Esfandiar Maasoumi, and Estela Bee Dagum. 2015. Entropy testing for nonlinear serial dependence in time series. Biometrika 102: 661-75. [CrossRef]

Granger, Clive WJ, and Namwon Hyung. 2004. Occasional structural breaks and long memory with an application to the S\&P 500 absolute stock returns. Journal of Empirical Finance 11: 399-421.

Granger, Clive WJ, Bwo-Nung Huangb, and Chin-Wei Yang. 2000. A bivariate causality between stock prices and exchange rates: evidence from recent Asianflu. The Quarterly Review of Economics and Finance 40: 337-54. [CrossRef]

Gulko, Les. 1999. The entropy theory of stock option pricing. International Journal of Theoretical and Applied Finance 2: 331-55. [CrossRef]

Katsiampa, Paraskevi. 2017. Volatility estimation for Bitcoin: A comparison of GARCH models. Economics Letters 158: 3-6. [CrossRef]

Kristoufek, Ladislav. 2015. What are the main drivers of the Bitcoin price? Evidence from wavelet coherence analysis. PLoS ONE 10: e0123923. [CrossRef] [PubMed]

Kullback, Solomon, and Richard A. Leibler. 1951. On information and sufficiency. The Annals of Mathematical Statistics 22: 79-86. [CrossRef]

Lahmiri, Salim, and Stelios Bekiros. 2019. Cryptocurrency forecasting with deep learning chaotic neural networks. Chaos, Solitons $\mathcal{E}$ Fractals 118: 35-40.

Lahmiri, Salim, and Stelios Bekiros. 2018. randomness and multi-fractality in Bitcoin market. Chaos, Solitons E Fractals 106: 28-34.

Li, Xin, and Chong Alex Wang. 2017. The technology and economic determinants of cryptocurrency exchange rates: The case of Bitcoin. Decision Support Systems 95: 49-60. [CrossRef]

Maasoumi, Esfandiar, and Jeff Racine. 2002. Entropy and predictability of stock market returns. Journal of Econometrics 107: 291-312. [CrossRef]

Naeem, Muhammad Abubakr, Saba Qureshi, Mobeen Ur Rehman, and Faruk Balli. 2021. COVID-19 and cryptocurrency market: Evidence from quantile connectedness. Applied Economics, 1-27. [CrossRef]

Nakayama, Ken, and Gerald H. Silverman. 1986. Serial and parallel processing of visual feature conjunctions. Nature 320: $264-65$. [CrossRef] [PubMed]

Osterrieder, Joerg, and Julian Lorenz. 2017. A statistical risk assessment of Bitcoin and its extreme tail behavior. Annals of Financial Economics 12: 1750003. [CrossRef]

Pichl, Lukas, and Taisei Kaizoji. 2017. Volatility analysis of bitcoin. Quantitative Finance and Economics 1: 474-85. [CrossRef]

Renyi, Alfred. 1961. On measures of entropy and information. In Proceedings of the Fourth Berkeley Symposium on Mathematical Statistics and Probability. Volume 1: Contributions to the Theory of Statistics. Oakland, California: University of California Press; pp. 547-61.

Renyi, Alfred. 1970. On the number of endpoints of a k-tree. Studia Scientiarum Mathematicarum Hungarica 5: 5-10. 
Sarkodie, Samuel Asumadu, Maruf Yakubu Ahmed, and Phebe Asantewaa Owusu. 2021. COVID-19 pandemic improves market signals of cryptocurrencies? evidence from Bitcoin, Bitcoin Cash, Ethereum, and Litecoin. Finance Research Letters: 102049. [CrossRef]

Shannon, Claude Elwood. 1948. A mathematical theory of communication. The Bell System Technical Journal 27: 379-423. [CrossRef] Skaug, Hans Julius, and Dag Tjostheim. 1996. Testing for serial independence using measures of distance between densities. In Athens Conference on Applied Probability and Time Series Analysis. New York: Springer; pp. 363-77.

Stavroyiannis, Stavros. 2018. Value-at-risk and related measures for the Bitcoin. The Journal of Risk Finance 19: 127-36. [CrossRef]

Thies, Sven, and Peter Molnar. 2018. Bayesian change point analysis of Bitcoin returns. Finance Research Letters 27: 223-27. [CrossRef]

Vukovic, Darko, Moinak Maiti, Zoran Grubisic, Elena M. Grigorieva, and Michael Frömmel. 2021. COVID-19 Pandemic: Is the Crypto Market a Safe Haven? The Impact of the First Wave. Sustainability 13: 8578. [CrossRef] 